\title{
Enriching UDDI Information Model with an Integrated Service Profile
}

\author{
Natenapa Sriharee and Twittie Senivongse \\ Department of Computer Engineering, Chulalongkorn University, \\ Phyathai Road, Pathumwan, Bangkok 10330 Thailand \\ natenapa.s@student.chula.ac.th, twittie.s@chula.ac.th
}

\begin{abstract}
Service discovery is one key aspect in the enabling technologies for service-oriented systems including Web Services. Growing attention has been paid to the content of business and service descriptions to allow services to be discovered more flexibly and accurately. This paper presents an information model called an integrated service profile that covers aspects of Web Services, i.e. attribute-based profile and specification-based profiles. Attribute-based profile contains attributes that are described more easily with some values, while specification-based profiles represent attributes whose values are complex and then should be described as specifications. The specifications could describe Web Services in terms of their semantic structure, behaviour and rules, and composition. The paper proposes to represent these specifications by an ontology language, and hence they can be used further to discover Web Services semantically.
\end{abstract}

\section{Introduction}

The diversity of the format and content of service descriptions within a serviceoriented environment has been problematic for service consumers when looking for available services. Standard UDDI registry for Web Services [1] attempts to standardise business and service descriptions through a set of business and service attributes. However, the attribute set is coarse and gives only preliminary information about the service providers and the offered Web Services. Generally a search is by matching of the name or category of Business Entities, Business Services, or tModels against the values specified in the query, such as "I want to find service providers in the electronics appliance category". The search will return some information and the rest is left to the service consumer to browse the Web pages of those companies to make a selection. The search does not yet support a query that is also based on semantic or behavioural information such as "I want to find an online electronics shop that sells desktop computers and is awarded Best Electronics Appliance Vendor from the Ministry of Commerce. The store should accept Amex credit card and deliver the computer that I have bought to my place (in Bangkok)".

It is assumed that service providers will do their best to please service consumers, and will try to advertise useful information as much as they can to facilitate the consumers as well as to get themselves discovered easily. Our work then started with the 
question "What should be in a service description to allow service consumers to query more conveniently and flexibly?" We started to survey on the service descriptions and their contents, and the result is an integrated service profile. This profile covers aspects of Web Services, i.e. attribute-based profile and specification-based profiles. Attribute-based profile contains attributes that are described more easily with some values, while specification-based profiles are associated with the attributes whose values are complex and then should be described as specifications. The specifications describe Web Services in terms of their semantic structure, behaviour and rules, and composition. This paper discusses the integrated service profile and reports some results of our study on defining and using the integrated profile.

Section 2 presents the attribute-based profile which is the result of the survey on the contents of service descriptions and Section 3 presents the specification-based profiles. Section 4 discusses the usage of the integrated service profile and some related work. The paper is concluded in Section 5.

\section{Attribute-Based Profile: Survey on Web Services Descriptions}

A survey was conducted to find in what way the description of a Web Service could be enriched. We gained some result from Web Services brokerage sites (such as www.capescience.com, www.webserviceoftheday.com, www.salcentral.com, www. xmethods.com), and we additionally looked further at the advertisements of software components on the Internet and at the literature on software components since software components and Web Services have similar characteristics (although there are different points) [2]. Considering both functional and psychological needs [3], the result of the survey is summarised in Fig. 1. (See [4] for details of the survey.) Most of the attributes here are optional, meaning that it is recommended to declare when applicable. The attributes that are shaded are not currently supported by standard UDDI but they can be imported by using specialised tModels that store the attribute values. Those that are not shaded are already supported by various parts of UDDI information model.

Most attributes in the attribute-based profile have values that are meaningful to service consumers (e.g. attribute Award) but those in the specification part of the profile represent more complex information that is hard to describe as attribute values. Therefore, each of these specification-based attributes may instead refer to (the URL of) the corresponding specification-based profile. We are interested in representing such profiles in a way that will enable a better service discovery, so we focus on ontology-based specifications (e.g. in OWL) for semantics-based discovery.

\section{Specification-Based Profiles}

Specification-based profiles will be derived from three upper ontologies (Fig. 2). The structural upper ontology, adapted from [5], represents semantic or knowledge structure of a Web Service. The behavioural upper ontology, corresponding to part of OWL-S service profile [6], represents service behaviour. The rule upper ontology represents a business rules policy or constraints that are put on the service behaviour. 


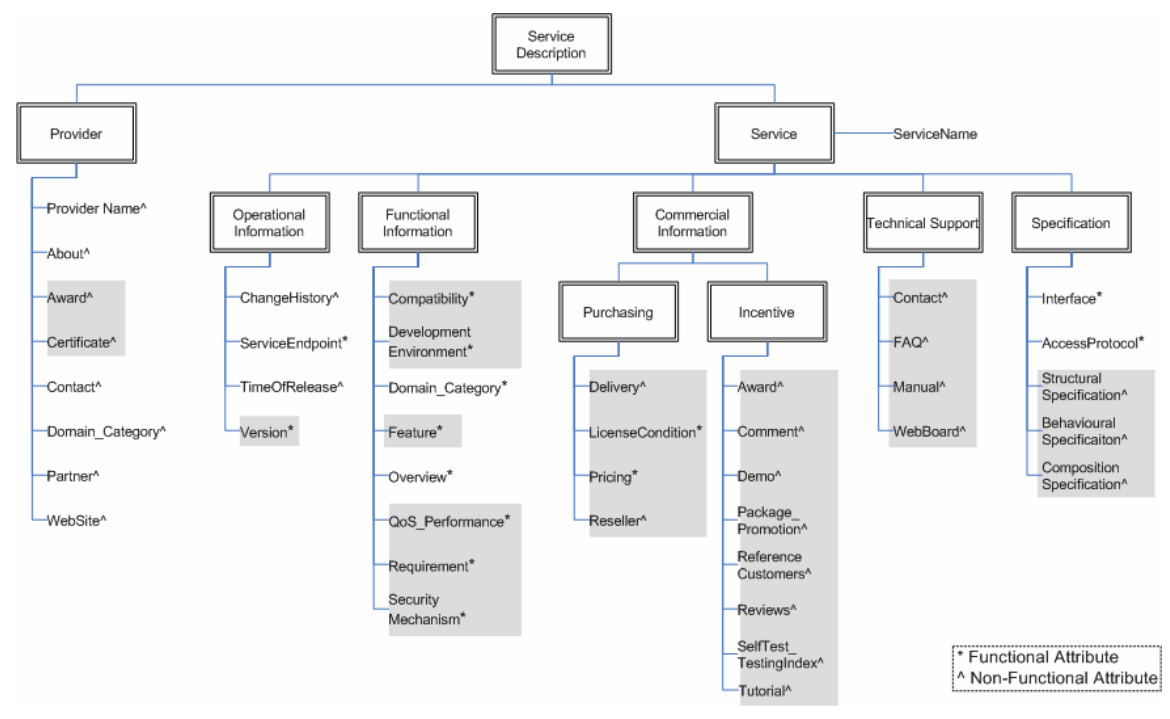

Fig. 1. Attribute-based profile

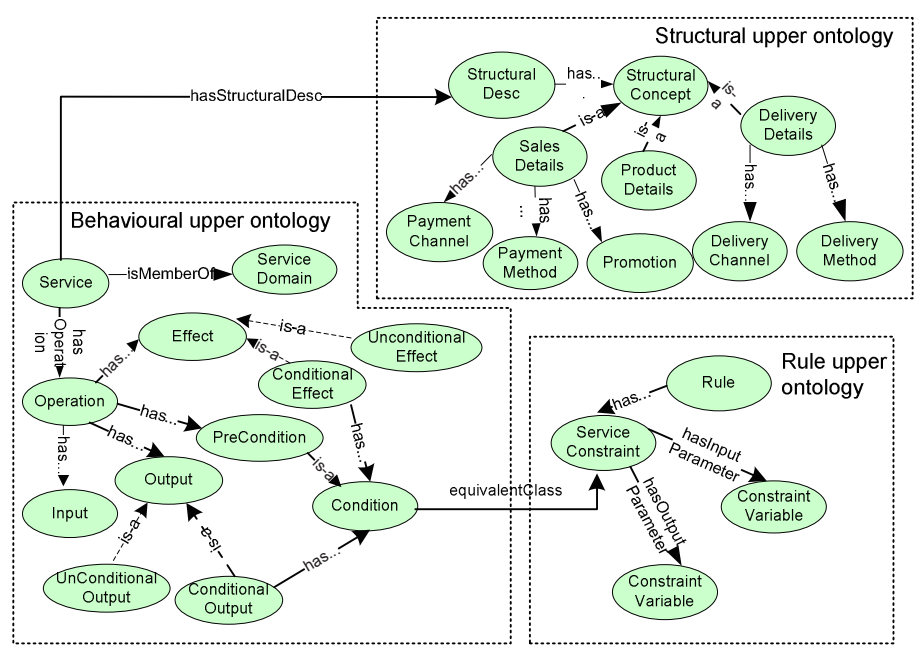

Fig. 2. Upper ontologies for specification-based profiles

From the upper ontologies, domain experts can derive corresponding domainspecific ontologies in order for the service providers to further derive their own profiles. Fig. 3(1) shows part of the structural ontology of the electronics appliance domain that is derived from the structural upper ontology. A service provider named PowerBuy then derives from this structural ontology to create its own structural profile in Fig. 3(2). Similarly, PowerBuy follows the behavioural ontology of the 


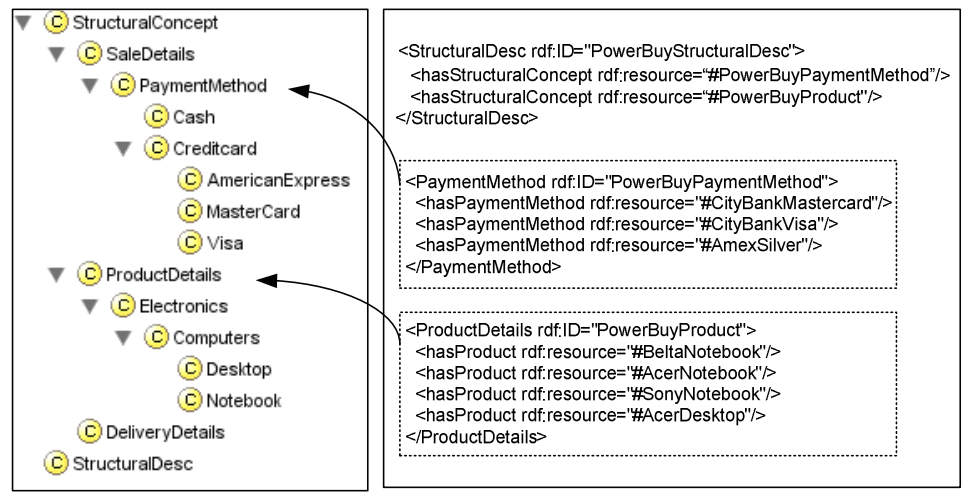

(1)

(2)

Fig. 3. Structural specification (1) Part of structural ontology for electronics appliance domain (2) Part of structural profile of PowerBuyEshop service

electronics appliance domain in Fig. 4(1) to define its own behavioural profile (Fig. $4(2)$ ). Note that PowerBuy has a condition ValidShippingLocation associated with its conditional effect - ProductDelivered. This means the product will be delivered only to some valid locations. Suppose that in this behavioural profile, ValidShippingLocation is defined as an equivalent class to the class ServiceShippingLocation which is in the rule ontology for the electronics appliance domain (Fig. 5(1)), PowerBuy can then create a rule profile for ServiceShippingLocation (Fig. 5(2)). The rule profile here refers to an associated rule definition, written in ABLE rule language (Fig. 5(3)). The rule definition says that the valid delivery locations are Bangkok and Rachaburi only.

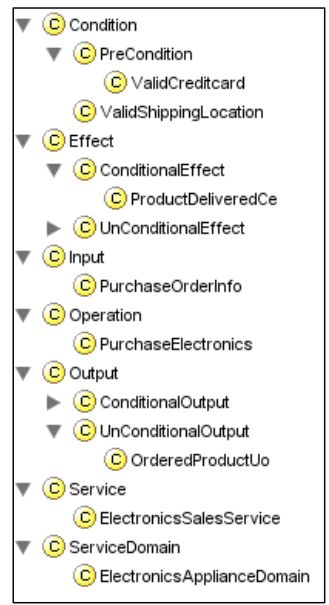

(1)

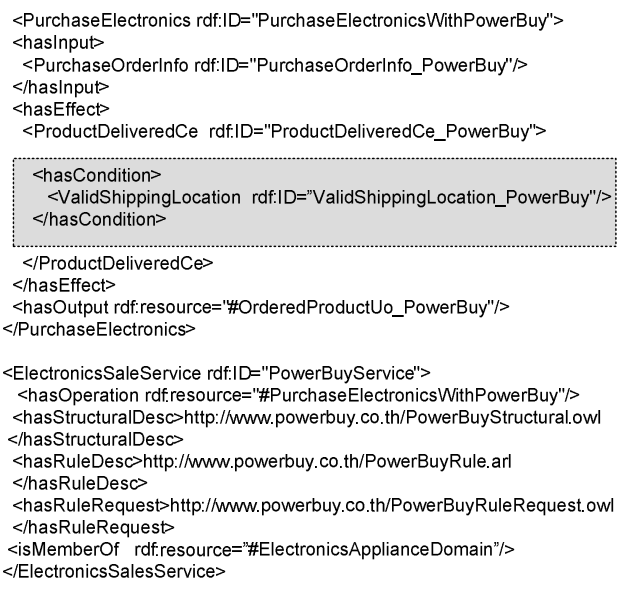

Fig. 4. Behavioural specification (1) Behavioural ontology for electronics appliance domain (2) Behavioural profile of PowerBuyEshop service 


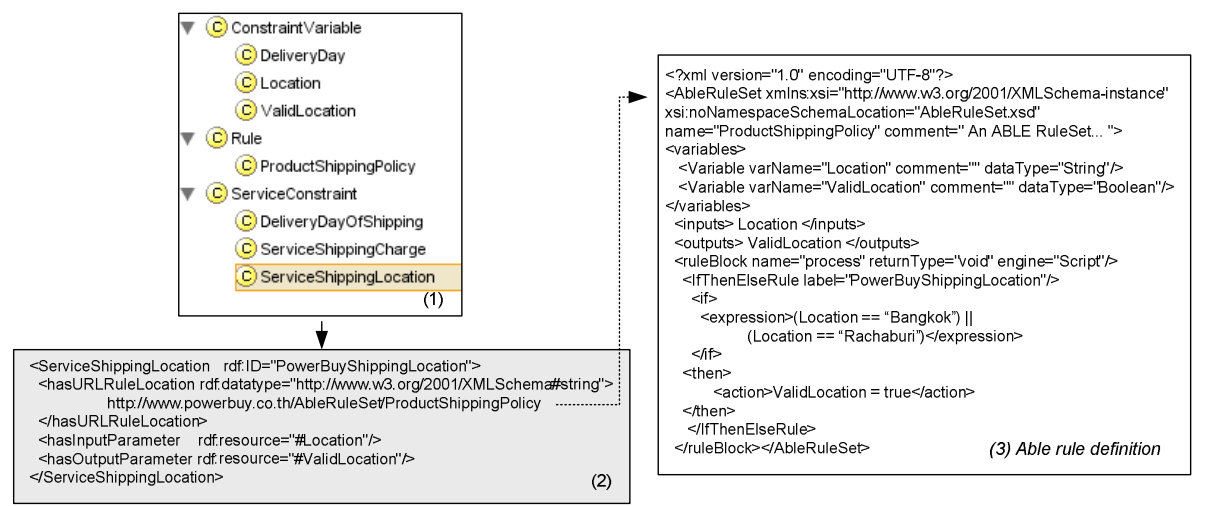

Fig. 5. Rule specification (1) Rule ontology of electronics appliance domain (2) Rule profile of PowerBuyEshop service (3) Rule definition in ABLE rule language

\section{Discussion}

With the integrated service profile, a service consumer can submit a semantic query such as the one mentioned in Section 1. The attribute-based profiles of the providers will be searched to find ones that are in the electronics appliance domain and have received the Best Electronics Appliance Vendor award. Then the structural profiles of such providers will be checked if they sell desktop computers, and their behavioural profiles are checked if they accepts Amex card and have a delivery service. The qualified candidates will have their rule profiles checked further to see if they can make a delivery to the consumer's address. By representing the semantics of Web Service with an ontology language, the query can also benefit from ontology reasoning. For example, the providers who advertise that they sell a PC would match this query for a desktop shop, or if they advertise their effect as ProductDeliveredWithDeliveryCharge, they would also match the query for ProductDelivered effect. Details of the matching algorithm and the discovery architecture that supports this integrated service profiles and query can be found in [7].

Research work that attempts to enhance Web Services discovery mostly introduces semantics for service descriptions and focuses on only one aspect of the semantics. For example, UDDI version 4 is trying to incorporate an ontology-based taxonomy for the standard categories of Business Entity and Business Service [8]. This effort will allow UDDI to also return businesses or services of a specialised or generalised category. The work in [5] focuses on the knowledge about the service and corresponds to the use of our structural profile. The work in [9][10] focuses on searching by service behaviour and corresponds to the use of our behavioural profile, but they do not consider using precondition and effect as the query constraints whereas we do. We consider our integrated service profile closest to OWL-S effort (especially the OWL-S Service Profile) in that a building block for rich semantic service descriptions is developed. The behavioural profile may overlap with a part of OWL-S Service Profile but it is enhanced by the use of the rule profile. Also, our 
attribute-based profile is more extensive than the attributes in OWL-S Service Profile as it is a compilation from an empirical survey.

\section{Conclusion}

The proposed integrated service profile is in accordance with the Service-Oriented Model part of the Web Services Architecture [11] in which a Web Service is modelled to have information about the provider, the syntax and semantics of the service, choreography of the tasks within the service, and a business policy. It allows service consumers to compose more complex and comprehensive queries.

We are in the process of completing the prototype of the discovery framework that integrates with the standard UDDI. We are researching on how to determine the degree of matching and will continue to explore discovery by composition specification.

\section{References}

1. uddi.org: UDDI: Universal Description, Discovery and Integration of Web Services (online). http://www.uddi.org

2. Yang, J.: Web Service Componentization. Communications of the ACM Vol. 46 No. 10. October (2003) 35-40

3. O'Sullivan, et al.: What's in a Service? Towards Accurate Description of Non-Functional Service Properties. Distributed and Parallel Databases Vol. 12 (2002) 117-133

4. Teppaboot, C.: Attribute-Based Description Model for Distributed Services. Master Thesis, Dept. of Computer Engineering, Chulalongkorn University (2004)

5. Trastour, D. et al.: A Semantic Web Approach to Service Description for Matchmaking of Services. In: Proceedings of the International Semantic Web Working Symposium (SWWS'01) (2001)

6. Martin, D. et al.: Bringing Semantics to Web Services: The OWL-S Approach. In: Proceedings of $1^{\text {st }}$ International Workshop on Semantic Web Services and Web Process Composition (SWSWPC 2004), July (2004)

7. Sriharee, N.: Towards Semantic Discovery of Web Services Using an Integrated Service Profile. Tech. Report, Dept. of Computer Engineering, Chulalongkorn University (2005)

8. Paolucci, M., Sycara, K.: UDDI Spec TC V4 Proposal Semantic Search (online). (2004). http://www.oasis-open.org/committees/uddi-spec/doc/req/uddi-spec-tc-req029semanticsearch-20040308.doc

9. Paolucci, M. et al.: Semantic Matching of Web Services Capabilities. Proceedings of the $1^{\text {st }}$ International Semantic Web Conference (ISWC 2002), Sardinia (Italy), Lecture Notes in Computer Science, Vol. 2342. Springer Verlag (2002)

10. Sivashanmugan, K., Verma, K., Sheth, A., Miller, J.: Adding Semantics to Web Services Standards. Proceedings of the International Conference on Web Services (2003)

11. W3C: Web Services Architecture (online). (2004). http://www.w3.org/TR/2004/NOTEws-arch-20040211/ 